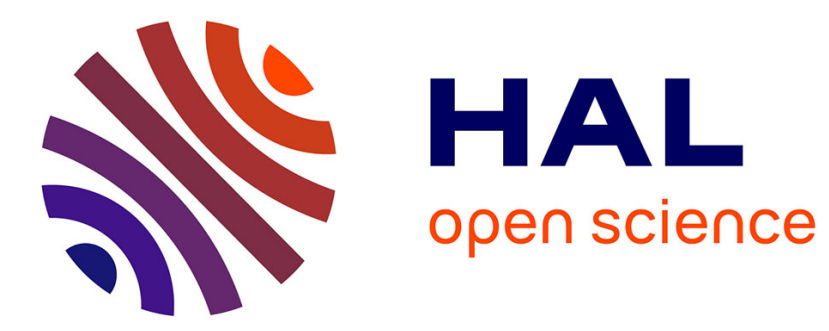

\title{
Salient and reentrant points of discrete sets
}

Alain Daurat, Maurice Nivat

\section{To cite this version:}

Alain Daurat, Maurice Nivat. Salient and reentrant points of discrete sets. Discrete Applied Mathematics, 2005, 151, pp.106-121. 10.1016/j.dam.2005.02.024 . hal-00023087

\section{HAL Id: hal-00023087 \\ https://hal.science/hal-00023087}

Submitted on 19 Apr 2006

HAL is a multi-disciplinary open access archive for the deposit and dissemination of scientific research documents, whether they are published or not. The documents may come from teaching and research institutions in France or abroad, or from public or private research centers.
L'archive ouverte pluridisciplinaire HAL, est destinée au dépôt et à la diffusion de documents scientifiques de niveau recherche, publiés ou non, émanant des établissements d'enseignement et de recherche français ou étrangers, des laboratoires publics ou privés. 


\title{
Salient and Reentrant Points of Discrete Sets
}

\author{
Alain Daurat ${ }^{a, 1}$ and Maurice Nivat ${ }^{b}$ \\ ${ }^{a}$ LSIIT CNRS UMR 7005, Université Louis Pasteur (Strasbourg 1), Pôle API, \\ Boulevard Sébastien Brant, 67400 Illkirch-Graffenstaden, France, \\ daurat@dpt-info.u-strasbg.fr \\ ${ }^{\mathrm{b}}$ LIAFA CNRS UMR 7089, Université Denis Diderot (Paris 7), Case 7014, \\ 2 place Jussieu, 75251 Paris Cedex 05, France, \\ Maurice.Nivat@liafa.jussieu.fr
}

\begin{abstract}
The border-salient and reentrant points of a discrete set are special points of the border of the set. When they are given with multiplicity they completely characterize the set, and without multiplicity they characterize the set if all its 8-components are 4-connected. The inner-salient and reentrant are defined similarly to the border ones, but we show that, in general, they do not characterize the set, even if this set is 4-simply connected. We also show that the genus of a set can be easily computed from the number of salient and reentrant points.
\end{abstract}

A discrete set is a finite subset of the integer plane $\mathbb{Z}^{2}$. Intuitively a discrete set can be described by its border, but this border can also be characterized by the points where there is a change of direction. In this paper these points are said to be salient and reentrant points. In fact we define two types of salient and reentrant points: the first ones are on the border of the set (the border points), and the second ones are in the inner of the set (the inner points).

Section 1 of this paper presents some preliminary definitions and properties. The section 2 is devoted to the border salient/reentrant points: we first prove that the genus of a set can be computed from the number of salients points and the number of reentrant points, it is in fact a reformulation of the well known result that the genus of a set can be locally computed. Then we prove that any discrete set is completely characterized by its set of border salient/reentrant points given with multiplicities. If the multiplicities are not given then the set must satisfy some connectivity constraints to be characterized.

$\overline{1}$ Partially supported by the Project EPML-9 of French CNRS. 
Section 3 presents the inner salient/reentrant points. We show that in general discrete sets are not characterized by their sets of inner salient/reentrant points even if the points are given with multiplicities and the sets are connected, nevertheless we show that there is a characterization theorem if the sets are supposed to satisfy convexity constraints.

\section{Preliminaries}

\subsection{Definitions and Notations}

A multiset is a set where each element can be repeated. If $E$ is a multiset, and $M \in E$ we denote by $\operatorname{mult}_{E}(M)$ the number of repetitions of $M$ in $E$. The set of elements of $E$ (where all the elements are counted only one time) is denoted $\operatorname{supp}(E)$. So, formally a multiset $E$ is the function mult $_{E}: F \rightarrow \mathbb{N}^{*}$ where $F=\operatorname{supp}(E)$.

The cardinal of a set $E$ is denoted $|E|$. If $E$ is a multiset then $|E|$ is the sum of the multiplicities of the elements of $E$. The complementary of a discrete set $E$ is the set $\mathbb{Z}^{2} \backslash E$ and is denoted $E^{c}$. The symmetrical difference of two sets $E$ and $F$ is $(E \backslash F) \cup(F \backslash E)$ and is denoted $E \Delta F$.

The half-integer plane denoted by $P_{1 / 2}$ is the image of the integer plane by the translation of vector $\left(\frac{1}{2}, \frac{1}{2}\right)$.

$$
P_{1 / 2}=\left\{\left(i+\frac{1}{2}, j+\frac{1}{2}\right):(i, j) \in \mathbb{Z}^{2}\right\}
$$

$([1])$.

A discrete set $E$ can be seen as a subset $C(E)$ of $\mathbb{R}^{2}$

$$
C(E)=\bigcup_{M \in E}\left(M+\left[-\frac{1}{2}, \frac{1}{2}\right]^{2}\right) .
$$

The border of $E$ is the topological boundary of $C(E)$ and is a finite union of polygonal closed lines whose edges are all horizontal or vertical and whose vertices are in $P_{1 / 2}$.

A 4-path (resp. an 8-path) is a finite sequence $\left(M_{0}, M_{1}, \ldots, M_{n}\right)$ of points of $\mathbb{Z}^{2}$ such that $M_{i+1}-M_{i}$ is in the set $\{( \pm 1,0),(0, \pm 1)\}$ (resp. $\left.\{( \pm 1,0),(0, \pm 1),( \pm 1, \pm 1)\}\right)$. Two points of $A$ and $B$ are said to be $k$-connected in a set $E \subset \mathbb{Z}^{2}(k \in\{4,8\})$ if there is a $k$-path between $A$ and $B$ and which is included in $E$. The $k$ connectedness is an equivalence relation on the points of $E$. The equivalence 
classes are the $k$-components of $E$. We denote by $n_{k}(E)$ the number of $k$ components of $E$. A set $E$ is said to be $k$-connected if it has only one $k$ component and it is said to be simply $k$-connected if $E$ is $k$-connected and $E^{c}$ is $(12-k)$-connected.

The lexicographical order on the points of $\mathbb{Z}^{2}$ is defined by:

$$
(x, y) \prec\left(x^{\prime}, y^{\prime}\right) \Leftrightarrow x<x^{\prime} \text { or }\left(x=x^{\prime} \text { and } y \leq y^{\prime}\right) \text {. }
$$

\subsection{Odd Border Points}

In this paragraph we define special points which will be very linked to the border-salient/reentrant points of next section.

Definition 1 If $E$ is a discrete set, the set of odd border points (denoted $\mathcal{O}(E)$ ) is :

$$
\mathcal{O}(E)=\left\{(i, j) \in P_{1 / 2}:\left|\left\{\left(i \pm \frac{1}{2}, j \pm \frac{1}{2}\right)\right\} \cap E\right| \in\{1,3\}\right\}
$$

The main interest of the definition is that a discrete set is completely characterized by its odd border points:

Proposition 2 If two discrete sets $E$ and $F$ satisfy $\mathcal{O}(E)=\mathcal{O}(F)$ then $E=$ $F$.

Lemma 3 Let $E$ be a discrete set and $M \notin E$ then $\mathcal{O}(E \cup\{M\})=\mathcal{O}(E) \Delta\left\{M+\left( \pm \frac{1}{2}, \pm \frac{1}{2}\right)\right\}$ where $\Delta$ denotes the symmetric difference.

PROOF. Let $N$ be in $\left\{M+\left( \pm \frac{1}{2}, \pm \frac{1}{2}\right)\right\}$. The point $M$ is not in $E$ so the number $\left|E \cap\left\{N+\left( \pm \frac{1}{2}, \pm \frac{1}{2}\right)\right\}\right|$ is odd if and only if $\left|(E \cup\{M\}) \cap\left\{N+\left( \pm \frac{1}{2}, \pm \frac{1}{2}\right)\right\}\right|$ is even. Then the lemma follows from the definition of $\mathcal{O}$.

PROOF of Proposition 2. We prove the property by recurrence on $|E|$. So we suppose that the property is true for any sets $E$ such that $|E| \leq n$ and we suppose that we have two sets $E$ and $F$ such that $|E|=n+1$ and $\mathcal{O}(E)=\mathcal{O}(F)$. Let $(x, y) \in \mathcal{O}(E)$ be the point of $P_{1 / 2}$ which is minimum for the lexicographical order. The point $M=\left(x+\frac{1}{2}, y+\frac{1}{2}\right)$ must be in both $E$ and $F$. So we consider $E^{\prime}=E \backslash\{M\}$ and $F^{\prime}=F \backslash\{M\}$. By the previous 
lemma:

$$
\begin{aligned}
\mathcal{O}\left(E^{\prime}\right) & =\mathcal{O}(E) \Delta\left\{M+\left( \pm \frac{1}{2}, \pm \frac{1}{2}\right)\right\} \\
& =\mathcal{O}(F) \Delta\left\{M+\left( \pm \frac{1}{2}, \pm \frac{1}{2}\right)\right\} \\
& =\mathcal{O}\left(F^{\prime}\right) .
\end{aligned}
$$

So by recurrence $E^{\prime}=F^{\prime}$ and then $E=F$.

\section{Border Salient/Reentrant Points}

\subsection{Definitions}

Definition $4 \cdot A$ corner is a couple $(M, N)$ when $M \in P_{1 / 2}$ and $N \in \mathbb{Z}^{2}$ and $M-N$ is in $\left\{\left( \pm \frac{1}{2}, \pm \frac{1}{2}\right)\right\}$.

- A corner $(M, N)$ is a salient corner of $E$ if $N \in E$ and $M$ is the extremity of two edges of the border of $E$ which are also sides of the square $N+\left[-\frac{1}{2}, \frac{1}{2}\right]^{2}$.

- A corner $(M, N)$ is a reentrant corner of $E$ if $N \in E$ and $M$ is the extremities of two consecutive edges of the border $E$ which are not sides of the square $N+\left[-\frac{1}{2}, \frac{1}{2}\right]^{2}$.

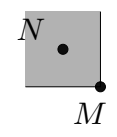

salient corner

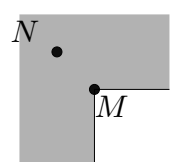

reentrant corner

Fig. 1. Corners

Now we can define the border-salient/reentrant points:

Definition 5 The multiset of border-salient (resp border-reentrant) points of a discrete set $E$, denoted $\mathcal{S}_{B}(E)$ (resp $\mathcal{R}_{B}(E)$ ) is the multiset whose support is included in $P_{1 / 2}$ and such that for any $M \in P_{1 / 2}$, the number mult $_{\mathcal{S}_{B}(E)}(M)$ (resp $\left.\operatorname{mult}_{\mathcal{R}_{B}(E)}(M)\right)$ is the number of $N$ such that $(M, N)$ is a salient corner (resp reentrant corner).

The different local configurations are described in figure 2. In particular a border-salient point can have multiplicity 1 or 2 , and a border-reentrant points has always the multiplicity 1 .

We notice that any odd border point is salient or reentrant. The inverse is not true since the salient points of multiplicity 2 are not odd border points. More 

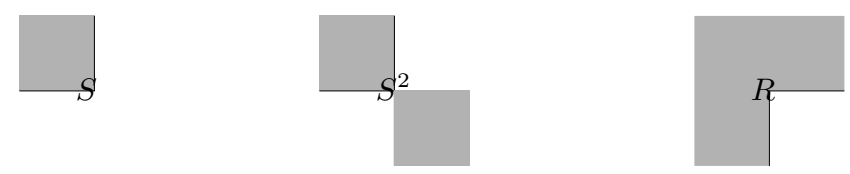

Fig. 2. Definition of the border salient and reentrant points. (The exponent indicates the multiplicity)

precisely if:

$$
\begin{aligned}
& \mathcal{D}(E)=\left\{(x, y) \in P_{1 / 2}: E \cap\left\{\left(x \pm \frac{1}{2}, y \pm \frac{1}{2}\right)\right\}=\right. \\
& \left.\left\{\left(x-\frac{1}{2}, y-\frac{1}{2}\right),\left(x+\frac{1}{2}, y+\frac{1}{2}\right)\right\} \text { or }\left\{\left(x-\frac{1}{2}, y+\frac{1}{2}\right),\left(x+\frac{1}{2}, y-\frac{1}{2}\right)\right\}\right\}
\end{aligned}
$$

then

$$
\begin{aligned}
& \mathcal{D}(E)=\left\{M \in \operatorname{supp}\left(\mathcal{S}_{B}(E)\right): \operatorname{mult}_{\mathcal{S}_{B}(E)}=2\right\} \\
& \mathcal{O}(E)=\left(\operatorname{supp}\left(\mathcal{S}_{B}(E)\right) \cup \mathcal{R}_{B}(E)\right) \backslash \mathcal{D}(E) .
\end{aligned}
$$

\subsection{Genus and number of salient/reentrant points}

We recall that the 4 -genus of a discrete set $E$ (denoted $\left.g_{4}(E)\right)$ is $n_{4}(E)-$ $n_{8}\left(E^{c}\right)+1$.

A first property of the salient and reentrant points is the computation of the genus with them:

Proposition 6 If $E$ is a discrete set then $g_{4}(E)=\frac{1}{4}\left(\left|\mathcal{S}_{B}(E)\right|-\left|\mathcal{R}_{B}(E)\right|\right)$. In particular if $E$ is 4-simply connected then $\left|\mathcal{S}_{B}(E)\right|=\left|\mathcal{R}_{B}(E)\right|+4$.

We recall that a 4 -simple point of $E$ is a point $M=(x, y) \in E$ such that $\{x \pm 1, y \pm 1\} \cap E$ is 4-connected and $\{x \pm 1, y \pm 1\} \cap E^{c}$ is 8-connected. A classical property of simple points is the following: ([4, Proposition 4.1])

Proposition 7 A point $M$ is a simple point of $E$ if and only if $n_{4}(E \backslash\{M\})=$ $n_{4}(E)$ and $n_{8}\left((E \backslash\{M\})^{c}\right)=n_{8}\left(E^{c}\right)$.

PROOF of Proposition 6. We will prove the property by recurrence on the cardinal of the set. Suppose that the property is true for any set $F$ such that $|F| \leq n-1$ and let $E$ be a set of cardinal $n$. Let $M=(x, y)$ be the point of $E$ which is minimum for the lexicographical order. We know that the set $E^{\prime}=E \backslash\{M\}$ satisfies $g_{4}\left(E^{\prime}\right)=\frac{1}{4}\left(\left|\mathcal{S}_{B}\left(E^{\prime}\right)\right|-\left|\mathcal{R}_{B}\left(E^{\prime}\right)\right|\right)$.

By hypothesis the points $(x-1, y-1),(x-1, y),(x-1, y-1),(x, y-1)$ are not in $E$, but the other four 8-neighborhoods of $M$ can be in $E$ or not, so there are 16 possibilities which are grouped in three cases (see Figure 3). 

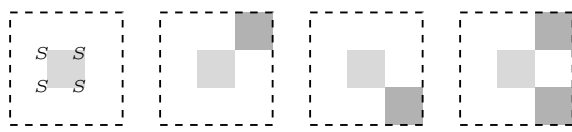

2.a
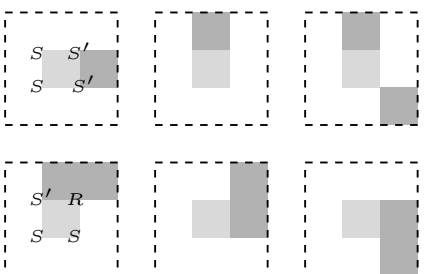

$\left|\mathcal{S}_{B}\left(E^{\prime}\right)\right|=\left|\mathcal{S}_{B}(E)\right|+1,\left|\mathcal{R}_{B}\left(E^{\prime}\right)\right|=\left|\mathcal{R}_{B}(E)\right|+1$,

$n_{4}\left(E^{\prime}\right)=n_{4}(E), n_{8}\left(E^{\prime c}\right)=n_{8}\left(E^{c}\right)$

$\left|\mathcal{S}_{B}\left(E^{\prime}\right)\right|=\left|\mathcal{S}_{B}(E)\right|-2,\left|\mathcal{R}_{B}\left(E^{\prime}\right)\right|=\left|\mathcal{R}_{B}(E)\right|-2$,

$n_{4}\left(E^{\prime}\right)=n_{4}(E), n_{8}\left(E^{\prime c}\right)=n_{8}\left(E^{c}\right)$

$\left|\mathcal{S}_{B}\left(E^{\prime}\right)\right|=\left|\mathcal{S}_{B}(E)\right|,\left|\mathcal{R}_{B}\left(E^{\prime}\right)\right|=\left|\mathcal{R}_{B}(E)\right|$,

$n_{4}\left(E^{\prime}\right)=n_{4}(E), n_{8}\left(E^{\prime c}\right)=n_{8}\left(E^{c}\right)$

$\left|\mathcal{S}_{B}\left(E^{\prime}\right)\right|=\left|\mathcal{S}_{B}(E)\right|+3,\left|\mathcal{R}_{B}\left(E^{\prime}\right)\right|=\left|\mathcal{R}_{B}(E)\right|-1$,

$n_{4}\left(E^{\prime}\right)=n_{4}(E)+1, n_{8}\left(E^{\prime c}\right)=n_{8}\left(E^{c}\right)$ or $n_{4}\left(E^{\prime}\right)=n_{4}(E), n_{8}\left(E^{\prime c}\right)=n_{8}\left(E^{c}\right)-1$

$\left|\mathcal{S}_{B}\left(E^{\prime}\right)\right|=\left|\mathcal{S}_{B}(E)\right|+2,\left|\mathcal{R}_{B}\left(E^{\prime}\right)\right|=\left|\mathcal{R}_{B}(E)\right|-2$,

$n_{4}\left(E^{\prime}\right)=n_{4}(E)+1, n_{8}\left(E^{\prime c}\right)=n_{8}\left(E^{c}\right)$ or $n_{4}\left(E^{\prime}\right)=n_{4}(E), n_{8}\left(E^{\prime c}\right)=n_{8}\left(E^{c}\right)-1$

Fig. 3. The different cases in the proof of Proposition 6 .

(1) In this case we suppose that the point $M$ is isolated, which means that the four points $(x \pm 1, y),(x, y \pm 1)$ are not in $E$. We have $S_{B}(E)=$ $S_{B}\left(E^{\prime}\right) \cup\left\{x \pm \frac{1}{2}, y \pm \frac{1}{2}\right\}$ and $R_{B}(E)=R_{B}\left(E^{\prime}\right)$. A 4-path in $E$ between two points of $E \backslash\{M\}$ cannot pass through $M$ since the four points $(x \pm 1, y \pm 1)$ are not in $E$, so $n_{4}(E)=n_{4}\left(E^{\prime}\right)+1$. An 8-path in $E^{\prime c}$ between two points $A, B$ can pass through $M$ but all the 8-neighborhood of $M$ which are in $E^{c}$ can be linked by an 8-path which does not pass through $M$, so we can modify the initial 8-path so that it does not pass through $M$ but always links $A$ and $B$ and is contained in $E^{c}$ thus $n_{8}\left(E^{c}\right)=n_{8}\left(E^{\prime c}\right)$. So finally

$$
\begin{aligned}
g_{4}(E) & =n_{4}(E)-n_{8}\left(E^{c}\right)+1 \\
& =n_{4}\left(E^{\prime}\right)+1-n_{8}\left(E^{\prime c}\right)+1 \\
& =g_{4}\left(E^{\prime}\right)+1 \\
& =\frac{1}{4}\left(\left|S_{B}\left(E^{\prime}\right)\right|-\left|R_{B}\left(E^{\prime}\right)\right|\right)+1 \quad \text { by the recurrence hypothesis } \\
& =\frac{1}{4}\left(\left|S_{B}(E)\right|-4-\left|R_{B}(E)\right|\right)+1 \\
& =\frac{1}{4}\left(\left|S_{B}(E)\right|-\left|R_{B}(E)\right|\right)
\end{aligned}
$$


(2) We now consider the case where $M$ is a simple point. By Proposition 7 we have $n_{4}(E)=n_{4}\left(E^{\prime}\right)$ and $n_{8}\left(E^{c}\right)=n_{8}\left(E^{\prime c}\right)$. By considering all the possibilities (cases 2. of Figure 3) we can see that $\left(\left|S_{B}(E)\right|-\left|R_{B}(E)\right|\right)=$ $\left|S_{B}\left(E^{\prime}\right)\right|-\left|R_{B}\left(E^{\prime}\right)\right|$ and so $g_{4}(E)=\frac{1}{4}\left(\left|S_{B}(E)\right|-\left|R_{B}(E)\right|\right)$.

(3) It remains the case where $M$ is neither simple nor isolated. By Proposition 7 we know that

$$
n_{4}(E) \neq n_{4}\left(E^{\prime}\right) \text { or } n_{8}\left(E^{c}\right) \neq n_{8}\left(E^{\prime c}\right) .
$$

Let $A=(x, y+1), B=(x+1, y), C=(x+1, y+1), D=(x-1, y-1)$. We have $A, B \in E$ and $C, D \in E^{c}$ (cases 3. of Figure 3). The 4-components of $E$ which do not contain neither $A$ nor $B$ are also 4-components of $E^{\prime}$, similarly the 8-components of $E^{c}$ which do not contain neither $C$ or $D$ are 8-components of $E^{\prime c}$. So

$$
n_{4}(E) \leq n_{4}\left(E^{\prime}\right) \leq n_{4}(E)+1 \text { and } n_{8}\left(E^{\prime c}\right) \leq n_{8}\left(E^{c}\right) \leq n_{8}\left(E^{\prime c}\right)+1 .
$$

If $A$ and $B$ are 4-connected in $E^{\prime}$, then we have $n_{4}(E)=n_{4}\left(E^{\prime}\right)$ and so by (3) and (4) we have $n_{8}\left(E^{c}\right)=n_{8}\left(E^{\prime c}\right)+1$. If $A$ and $B$ are not 4-connected in $E^{\prime}$ then by considering the border of the 4-component of $E^{\prime}$ containing $A$ we can prove that $C$ and $D$ are 8-connected in $E^{c}$ (see border following algorithm $B F_{4}$ in [4, p626]). Then $n_{4}(E)=n_{4}\left(E^{\prime}\right)-1$ and $n_{8}\left(E^{c}\right)=$ $n_{8}\left(E^{\prime c}\right)$. So in the two cases we have $n_{4}(E)-n_{8}\left(E^{c}\right)=n_{4}\left(E^{\prime}\right)-n_{8}\left(E^{\prime c}\right)-1$. Moreover we have $\left|\mathcal{S}_{B}\left(E^{\prime}\right)\right|-\left|\mathcal{R}_{B}\left(E^{\prime}\right)\right|=\left|\mathcal{S}_{B}(E)\right|-\left|R_{B}(E)\right|+4$ (see the two cases 3.a, 3.b of Figure 3). So $g_{4}(E)=\frac{1}{4}\left(\left|S_{B}(E)\right|-\left|R_{B}(E)\right|\right)$.

Remark 8 In [5, Proposition 2.7.1] it is proved that $g_{4}(E)=|E|-e_{1}-e_{2}+f$ with:

$$
\begin{aligned}
e_{1} & =\mid\{(x, y):|E \cap\{(x, y),(x+1, y)\}|=2\}, \\
e_{2} & =\mid\{(x, y):|E \cap\{(x, y),(x, y+1)\}|=2\}, \\
f & =\mid\{(x, y):|E \cap\{(x, y),(x+1, y),(x, y+1),(x+1, y+1)\}|=4\} .
\end{aligned}
$$

In fact the proofs of these two propositions are very similar. Moreover we can deduce one from each other: Let $k_{1}, \ldots, k_{16}$ be the number of occurrences of each configuration in Figure 4 (notice that $k_{1}=\infty$ and the other $k_{i}$ are finite). By considering the configurations which have a common bar we have:

$$
\begin{gathered}
k_{2}+k_{6}+k_{10}+k_{14}=k_{5}+k_{6}+k_{7}+k_{8}, \\
k_{3}+k_{7}+k_{11}+k_{15}=k_{9}+k_{10}+k_{11}+k_{12}, \\
k_{4}+k_{8}+k_{12}+k_{16}=k_{13}+k_{14}+k_{15}+k_{16}, \\
k_{2}+k_{4}+k_{10}+k_{12}=k_{3}+k_{4}+k_{7}+k_{8}, \\
k_{5}+k_{7}+k_{13}+k_{15}=k_{9}+k_{10}+k_{13}+k_{14}, \\
k_{6}+k_{8}+k_{14}+k_{16}=k_{11}+k_{12}+k_{15}+k_{16} .
\end{gathered}
$$




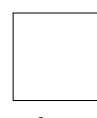

$k_{1}$

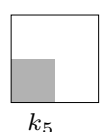

$k_{5}$

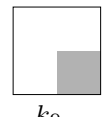

$k_{9}$

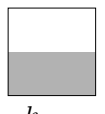

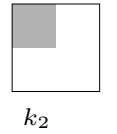
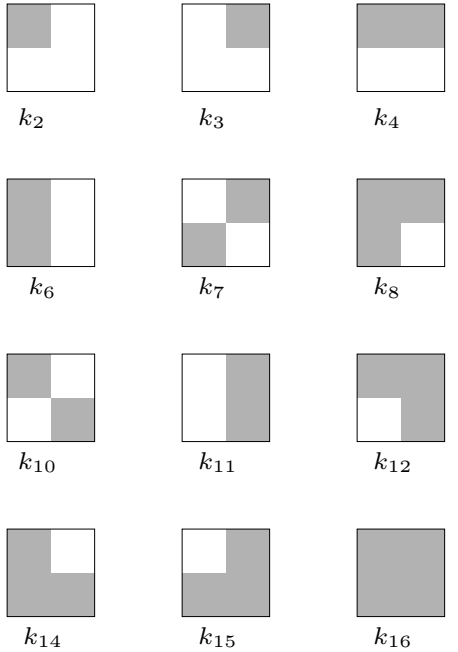

Fig. 4. The possibilities for the intersection of a discrete set and a $2 \times 2$ square.

We deduce that:

$$
\begin{aligned}
k_{10} & =k_{5}+k_{7}+k_{15}-k_{9}-k_{14}, \\
k_{4} & =-k_{8}-k_{3}+k_{5}+k_{13}+k_{15}, \\
k_{12} & =k_{3}+k_{14}-k_{5}, \\
k_{6} & =-k_{8}+k_{11}+k_{3}-k_{5}+k_{15}, \\
k_{2} & =-k_{15}+k_{9}+k_{8} .
\end{aligned}
$$

We have:

$$
\begin{aligned}
|E| & =k_{2}+k_{4}+k_{6}+k_{8}+k_{10}+k_{12}+k_{14}+k_{16}, \\
e_{1} & =k_{4}+k_{8}+k_{12}+k_{16}, \\
e_{2} & =k_{6}+k_{8}+k_{14}+k_{16}, \\
f & =k_{16}, \\
\left|\mathcal{S}_{B}(E)\right| & =k_{2}+k_{3}+k_{5}+k_{9}+2\left(k_{7}+k_{10}\right), \\
\left|\mathcal{R}_{B}(E)\right| & =k_{8}+k_{12}+k_{14}+k_{15}
\end{aligned}
$$

By combining (5) and (6) we have:

$$
\begin{aligned}
\left|\mathcal{S}_{B}(E)\right|-\left|\mathcal{R}_{B}(E)\right| & =-4 k_{15}+4 k_{9}+4 k_{10} \\
|E|-e_{1}-e_{2}+f & =k_{10}+k_{9}-k_{15} .
\end{aligned}
$$

and so $\frac{1}{4}\left(\left|S_{B}(E)\right|-\left|R_{B}(E)\right|=|E|-e_{1}-e_{2}+f\right.$.

See also [6, exercise G.4.c] for another similar property to Proposition 6 .

\subsection{Characterization of a set by its salient/reentrant points}

A direct consequence of Proposition 2 and Formula (2) is: 
Proposition 9 Let $E$ and $F$ be two discrete sets. If $\mathcal{S}_{B}(E)=\mathcal{S}_{B}(F)$ and $\mathcal{R}_{B}(E)=\mathcal{R}_{B}(F)$ then $E=F$.

Figure 5 shows that this proposition becomes wrong if $\mathcal{S}_{B}(E)$ is given without multiplicity. Nevertheless the two next propositions show that there is also a characterization property if we make supplementary hypothesis on the sets:

Proposition 10 If $n_{4}(E)=n_{8}(E), n_{4}\left(E^{c}\right)=n_{8}\left(E^{c}\right), n_{4}(F)=n_{8}(F), n_{4}\left(F^{c}\right)=$ $n_{8}\left(F^{c}\right)$ and $\operatorname{supp}\left(\mathcal{S}_{B}(E)\right) \cup \operatorname{supp}\left(\mathcal{R}_{B}(E)\right)=\operatorname{supp}\left(\mathcal{S}_{B}(F)\right) \cup \operatorname{supp}\left(\mathcal{R}_{B}(F)\right)$ then $E=F$.

Lemma 11 If $n_{4}(E)=n_{8}(E)$ and $n_{4}\left(E^{c}\right)=n_{8}\left(E^{c}\right)$ and $\mathcal{D}(E)$ is defined by (1) then $\mathcal{D}(E)$ is empty.

PROOF. Consider $L \in \mathbb{N}$ such that $E \subset[-(L-1),(L-1)]^{2}$, and $F=$ $[-L, L]^{2} \backslash E$.

$$
\begin{aligned}
g_{4}(E) & =n_{4}(E)-n_{8}\left(E^{c}\right)+1 \\
g_{4}(F) & =n_{4}(F)-n_{8}\left(F^{c}\right)+1 \\
& =n_{4}\left(E^{c}\right)-\left(n_{8}(E)+1\right)+1
\end{aligned}
$$

We have $n_{4}(E)=n_{8}(E)$ and $n_{4}\left(E^{c}\right)=n_{8}\left(E^{c}\right)$ so

$$
g_{4}(E)+g_{4}(F)=1
$$

We have $\left|\mathcal{S}_{B}(E)\right|=\left|\mathcal{R}_{B}(F)\right|+2|\mathcal{D}|$ and $\left|\mathcal{S}_{B}(F)\right|=\left|\mathcal{R}_{B}(E)\right|+2|\mathcal{D}|+4$. So

$$
\begin{aligned}
|\mathcal{D}(E)| & =\frac{\left|\mathcal{S}_{B}(E)\right|-\left|\mathcal{R}_{B}(F)\right|+\left|\mathcal{S}_{B}(F)\right|-\left|\mathcal{R}_{B}(E)\right|-4}{4} & & \text { by Formula }(2) \\
& =\frac{4 g_{4}(E)+4 g_{4}(F)-4}{4} & & \text { by Proposition } 6 \\
& =0 & & \text { by Formula ( } 7) .
\end{aligned}
$$

PROOF of Proposition 10. By Lemma 11, $\mathcal{D}(E)=\mathcal{D}(F)=\emptyset$, so $\mathcal{O}(E)=$ $\operatorname{supp}\left(\mathcal{S}_{B}(E)\right) \cup \mathcal{R}_{B}(E)=\operatorname{supp}\left(\mathcal{S}_{B}(F)\right) \cup \mathcal{R}_{B}(F)=\mathcal{O}(F)$ and the result follows from Proposition 6

Proposition 12 If $n_{4}(E)=n_{8}(E), n_{4}(F)=n_{8}(F), \operatorname{supp}\left(\mathcal{S}_{B}(E)\right)=\operatorname{supp}\left(\mathcal{S}_{B}(F)\right)$, $\operatorname{supp}\left(\mathcal{R}_{B}(E)\right)=\operatorname{supp}\left(\mathcal{R}_{B}(F)\right)$ then $E=F$.

PROOF. We take the abbreviations $S=\operatorname{supp}\left(\mathcal{S}_{B}(E)\right)=\operatorname{supp}\left(\mathcal{S}_{B}(F)\right)$ and $R=\operatorname{supp}\left(\mathcal{R}_{B}(E)\right)=\operatorname{supp}\left(\mathcal{R}_{B}(F)\right)$. We will construct the border of $E$ from 
$S$ and $R$. Precisely we will construct a sequence $\left(B_{i}^{k}\right)_{\substack{0 \leq k \leq n-1 \\ 0 \leq i \leq l_{k}-1}}$ of points of $P_{1 / 2}$ such that for a fixed $k$ the sequence $B^{k}=\left(B_{i}^{k}\right)_{0 \leq i \leq l_{k}-1}$ is the sequence of consecutive vertices of one of the polygonal closed lines of the border of $E$.

Suppose that we have constructed the sequence $B^{0}, B^{1}, \ldots, B^{k-1}$. If $S \cup R=$ $\left\{B_{i}^{k}: 0 \leq k \leq k-1\right.$ and $\left.0 \leq i \leq l_{k}-1\right\}$ then the integer $n$ is defined to be $k$. Otherwise we define $B_{0}^{k}$ as the minimum point $(x, y)$ for the lexicographical order of the set $(S \cup R) \backslash\left\{B_{i}^{k}: 0 \leq k \leq k-1\right.$ and $\left.0 \leq i \leq l_{k}-1\right\}$. The symbol $S^{\prime}$ will denote one of the set $S$ and $R$ which contains $B_{0}^{k}$ and $R^{\prime}$ will denote the other set. Now we define the sequence $B^{k}$ by a recurrence using an auxiliary sequence of vectors $\left(u_{i}\right)$. Intuitively the points of the sequence $\left(B^{k}\right)$ follow a mobile point which goes in the direction $u_{i}$. When this mobile point meets a salient point it turns 90 degrees on one side and when it meets a reentrant point it turns 90 degrees on the other side. Formally we have $u_{0}=\left(\frac{1}{2}, 0\right)$ and the following recurrence equation:

$$
\begin{aligned}
B_{i+1}^{k} & =B_{i}^{k}+t u_{i} & & \\
u_{i+1} & =r\left(u_{i}\right) & & \text { if } B_{i}^{k} \in S^{\prime} \\
& =r^{-1}\left(u_{i}\right) & & \text { if } B_{i}^{k} \in R^{\prime}
\end{aligned}
$$

where $t=\min \left\{t>0: B_{i}^{k}+t u_{i} \in S \cup R\right\}, r(x, y)=(y,-x), r^{-1}(x, y)=$ $(-y, x)$. The number $l_{k}$ is defined to be $\min \left\{i>0: B_{i}^{k}=B_{0}^{k}\right\}$. We can see by recurrence on $i$ that $\left(B_{i+1}^{k}, B_{i+1}^{k}-u_{i}+u_{i+1}\right)$ is a salient corner if $B_{i+1}^{k} \in S$ and $\left(B_{i+1}^{k}, B_{i+1}^{k}-u_{i}-u_{i+1}\right)$ is a reentrant corner if $B_{i+1}^{k} \in R$. By definition there is no salient or reentrant point in each segment $\left[B_{i}^{k}, B_{i+1}^{k}\right]$ so the polygonal closed line $\left(B_{0}^{k}, B_{1}^{k}, \ldots, B_{l_{k}-1}^{k}, B_{0}^{k}\right)$ is a component of the border of $E$.

So at the end of this process we have $n$ components $B^{0}, B^{1}, \ldots, B^{n-1}$ of the border of $E$, whose elements cover all $S \cup R$. If there was another component in the border of $E$, then a vertex $M$ of such component would belong to two polygonal lines. So $M$ must be a salient point with multiplicity 2 . Then $E \cap\left\{M+\left( \pm \frac{1}{2}, \pm \frac{1}{2}\right)\right\}=\{A, B\}$ where $A=M+\left(-\frac{1}{2},-\frac{1}{2}\right), B=M+\left(\frac{1}{2}, \frac{1}{2}\right)$ or $A=M+\left(-\frac{1}{2}, \frac{1}{2}\right), B=M+\left(\frac{1}{2},-\frac{1}{2}\right)$. Then $A$ and $B$ would not be not 4-connected in $E$, but they are 8-connected so we would have $n_{4}(E) \neq n_{8}(E)$. So the border of $E$ is the union of the polygonal lines $B^{0}, B^{1}, \ldots, B^{n-1}$, but it depends only on $S$ and $R$ so it is equal to the border of $F$ and then $E=F$. 

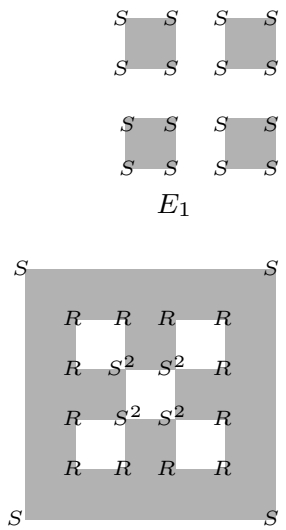

$E_{2}$

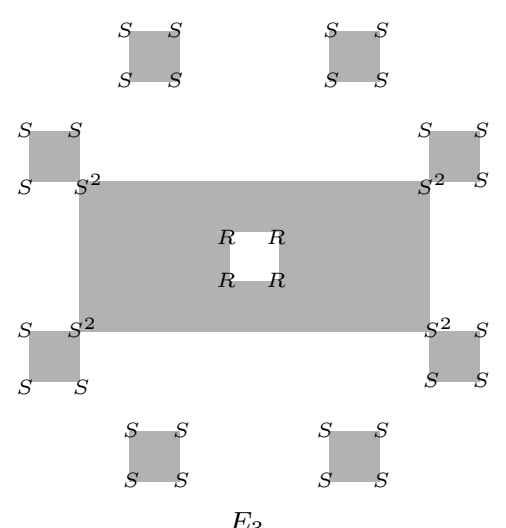

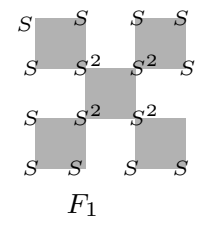

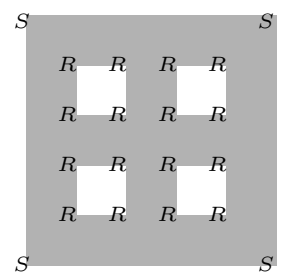

$F_{2}$

$$
\begin{aligned}
& \operatorname{supp}\left(\mathcal{S}_{B}\left(E_{1}\right)\right)=\operatorname{supp}\left(\mathcal{S}_{B}\left(F_{1}\right)\right) \\
& \operatorname{supp}\left(\mathcal{R}_{B}\left(E_{1}\right)\right)=\operatorname{supp}\left(\mathcal{R}_{B}\left(F_{1}\right)\right) \\
& \text { but } n_{4}\left(F_{1}\right) \neq n_{8}\left(F_{1}\right)
\end{aligned}
$$

$\operatorname{supp}\left(\mathcal{S}_{B}\left(E_{2}\right)\right) \cup \operatorname{supp}\left(\mathcal{R}_{B}\left(E_{2}\right)\right)=$ $\operatorname{supp}\left(\mathcal{S}_{B}\left(E_{2}\right)\right) \cup \operatorname{supp}\left(\mathcal{R}_{B}\left(E_{2}\right)\right)$ but $n_{4}\left(E_{2}^{c}\right) \neq n_{8}\left(E_{2}^{c}\right)$

Fig. 5. Pairs of sets which have near border salient/reentrant points.

\section{Inner Salient/Reentrant Points}

\subsection{General sets}

Definition 13 The multiset of inner-salient (resp inner-reentrant) points of a discrete set $E$, denoted $\mathcal{S}_{I}(E)$ (resp $\mathcal{R}_{I}(E)$ ) is the multiset whose support is included in $\mathbb{Z}^{2}$ and such that for any $N \in \mathbb{Z}^{2}$ the number mult ${ }_{\mathcal{S}_{I}(E)}(N)$ (resp mult $_{\mathcal{R}_{I}(E)}(N)$ ) is the number of $M$ such that $(M, N)$ is a salient corner (resp reentrant corner).

Figure 6 shows the different local configurations.

Proposition 14 If $E$ is a discrete set then $\left|\mathcal{S}_{I}(E)\right|=\left|\mathcal{S}_{B}(E)\right|,\left|\mathcal{R}_{I}(E)\right|=$ $\left|\mathcal{R}_{B}(E)\right|$.

PROOF. The multisets $\mathcal{S}_{I}(E)$ and $\mathcal{S}_{B}(E)$ are in bijection with the set of salient corners of $E$. In the same way the multisets $\mathcal{R}_{I}(E)$ and $\mathcal{R}_{B}(E)$ are in 

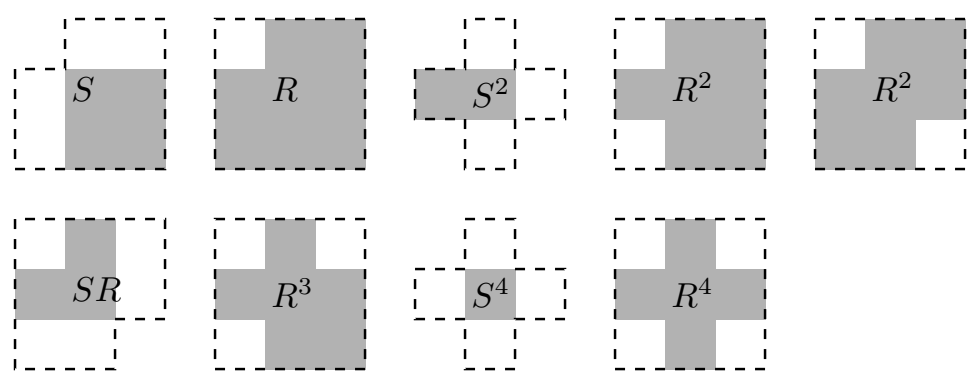

Fig. 6. Inner-salient/reentrant points. (The points outside the dashed boundary can be in $E$ or $E^{c}$ )

bijection with the set of reentrant corners of $E$.

In particular the formula computing the genus is also true for inner salient/reentrant points: $g_{4}(E)=\frac{1}{4}\left(\left|\mathcal{S}_{I}(E)\right|-\left|\mathcal{R}_{I}(E)\right|\right)$.

The inner salient/reentrant points do not determine the discrete sets even for the 4-simply connected sets (see figure 7).
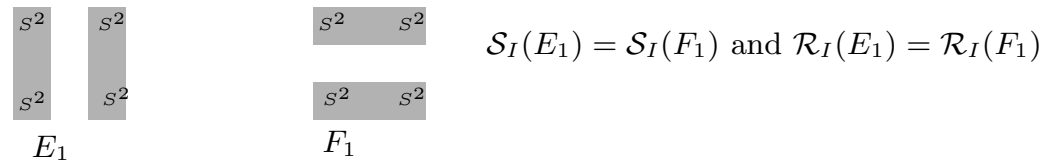

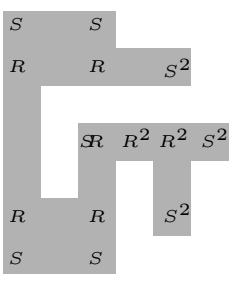

$E_{2}$

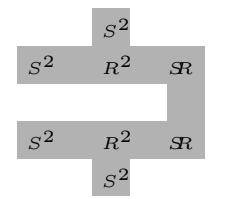

$E_{3}$

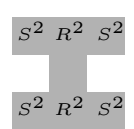

$E_{4}$

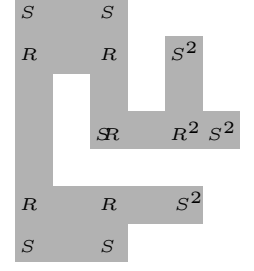

$F_{2}$ $\mathcal{S}_{I}\left(E_{2}\right)=\mathcal{S}_{I}\left(F_{2}\right)$ and $\mathcal{R}_{I}\left(E_{2}\right)=\mathcal{R}_{I}\left(F_{2}\right)$

$s^{2}$
$s^{2}$

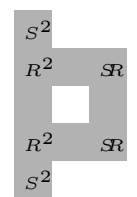

$F_{3}$

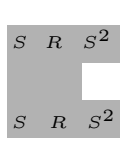

$E_{4}$
$\mathcal{S}_{I}\left(E_{3}\right)=\mathcal{S}_{I}\left(F_{3}\right)$ and $\mathcal{R}_{I}\left(E_{3}\right)=\mathcal{R}_{I}\left(F_{3}\right)$

$E_{3}$ is H-convex and 4-connected but

$F_{3}$ is not $\mathrm{H}$-convex

$\operatorname{supp}\left(\mathcal{S}_{I}\left(E_{4}\right)\right)=\operatorname{supp}\left(\mathcal{S}_{I}\left(F_{4}\right)\right)$ and $\operatorname{supp}\left(\mathcal{R}_{I}\left(E_{4}\right)\right)=\operatorname{supp}\left(\mathcal{R}_{I}\left(F_{4}\right)\right)$

$E_{4}$ and $F_{4}$ are H-convex and 4 -connected but they are not $\mathrm{V}$-convex.

Fig. 7. Pairs of sets which have near inner salient/reentrant points. 
Nevertheless, given two multisets $S$ and $R$ we can look for all the sets $E$ such that $\mathcal{S}_{I}(E)=S$ and $\mathcal{R}_{I}(E)=R$. Algorithm 1 does this, but restrictively for the class of the 4-simply connected sets. The idea of the algorithm is to find the border of the set, following the ideas of the proof of Proposition 12: we begin with the most left-bottom point of $S$ and we draw the border of the set by consider a mobile point which can turns left when it meets a salient point on its left, and can turns right when it meets a reentrant point. The problem is that sometimes there can be many possibilities for the mobile point (see figure 8). This case did not happen for border salient/reentrant points because the turn was always made at the first met salient/reentrant point. Algorithm 1 simply considers all the possibilities, so in fact it has an exponential complexity even if there is only one solution (see example of figure 9). We do not know if the construction of a discrete set from its salient/reentrant points can be done in polynomial time.

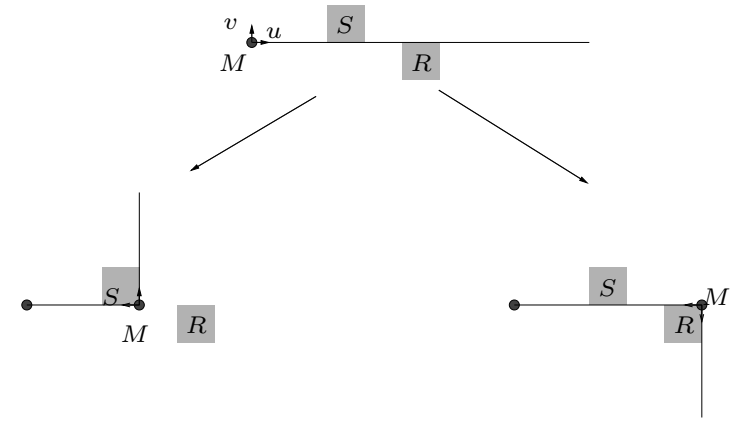

Fig. 8. The choices in Algorithm 1.

\subsection{Characterization of H-convex sets by Inner Salient Reentrant Points}

In this section and the following one, we will see that discrete sets can be determined by their salient/reentrants points if we impose some convexity constraints.

Definition 15 A set is H-convex (resp. V-convex) if the intersection of the set with any horizontal (resp. vertical) line is 4-connected. A discrete set is $\mathrm{HV}$-convex if it is H-convex and $V$-convex.

Proposition 16 Let $E$ and $F$ two $H$-convex sets. If $\mathcal{S}_{I}(E)=\mathcal{S}_{I}(F)$ and $\mathcal{R}_{I}(E)=\mathcal{R}_{I}(F)$ then $E=F$.

PROOF. Let $E$ be a H-convex set. For any $y \in \mathbb{Z}$ we define $x_{\min }(y)=$ $\min \{x:(x, y) \in E\}$ and $x_{\max }(y)=\max \{x:(x, y) \in E\}$ with the conventions $\min \varnothing=+\infty$ and $\max \varnothing=-\infty$. The set $E$ is H-convex so it is completely determined by the functions $x_{\min }$ and $x_{\max }$. For any $y$ we define 


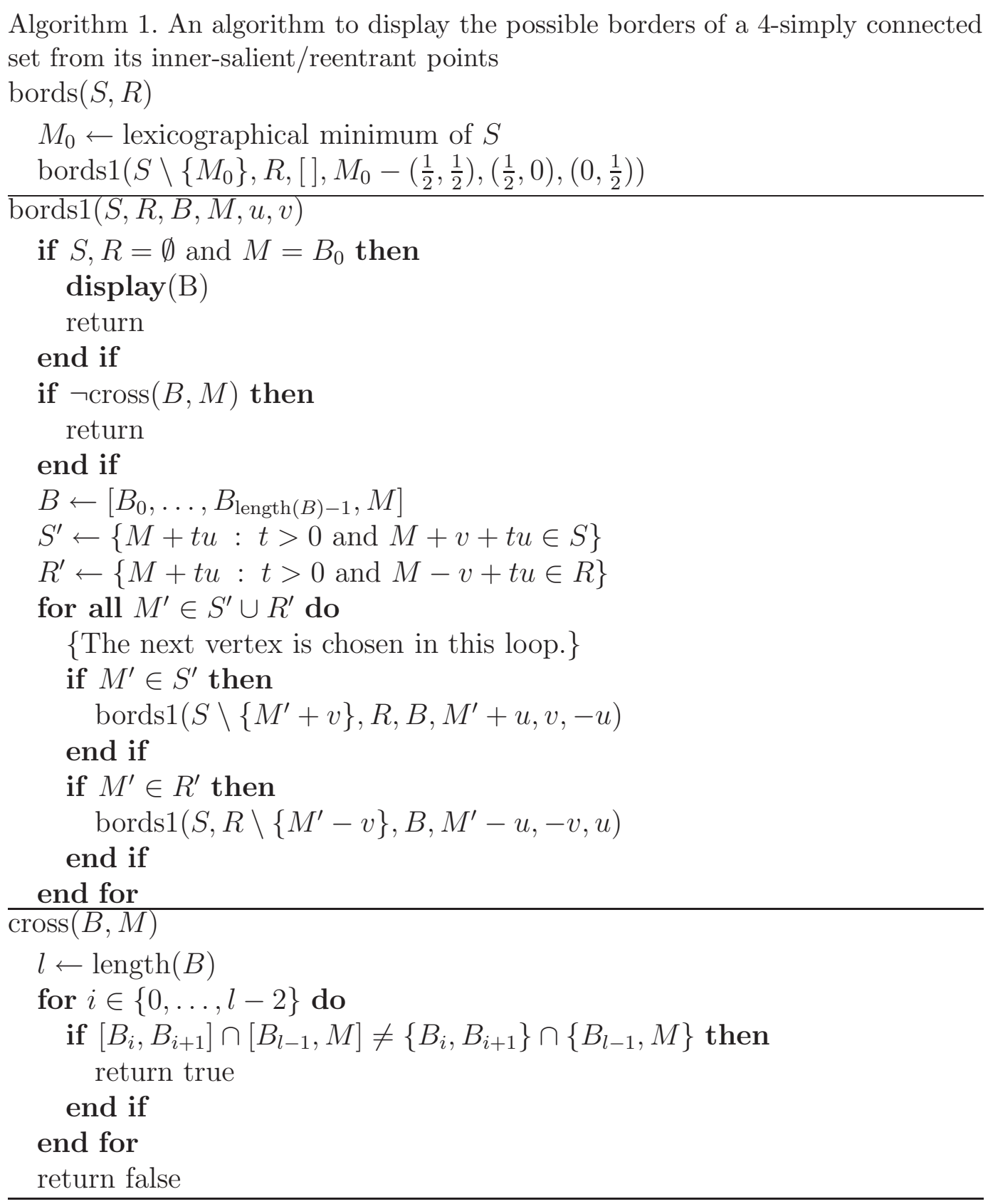

$S(y), R(y), S U(y), R U(y)$ as the multisets of integers defined by:

$$
\begin{aligned}
\operatorname{mult}_{S(y)}(x) & =\operatorname{mult}_{\mathcal{S}_{I}(E)}(x, y) \\
\operatorname{mult}_{R(y)}(x) & =\operatorname{mult}_{\mathcal{R}_{I}(E)}(x, y)
\end{aligned}
$$

$\operatorname{mult}_{S U(y)}(x)=\operatorname{mult}_{\mathcal{S}_{I}(E)}(x, y)-$

$$
\mid\left\{x^{\prime} \in\left\{x-\frac{1}{2}, x+\frac{1}{2}\right\}:\left(\left(x^{\prime}, y^{\prime}-\frac{1}{2}\right),(x, y)\right) \text { is a salient corner of } E\right\} \mid
$$

$\operatorname{mult}_{R U(x y)}(x)=\operatorname{mult}_{\mathcal{R}_{I}(E)}(x, y)-$

$$
\mid\left\{x^{\prime} \in\left\{x-\frac{1}{2}, x+\frac{1}{2}\right\}:\left(\left(x^{\prime}, y^{\prime}-\frac{1}{2}\right),(x, y)\right) \text { is a reentrant corner of } E\right\} \mid
$$



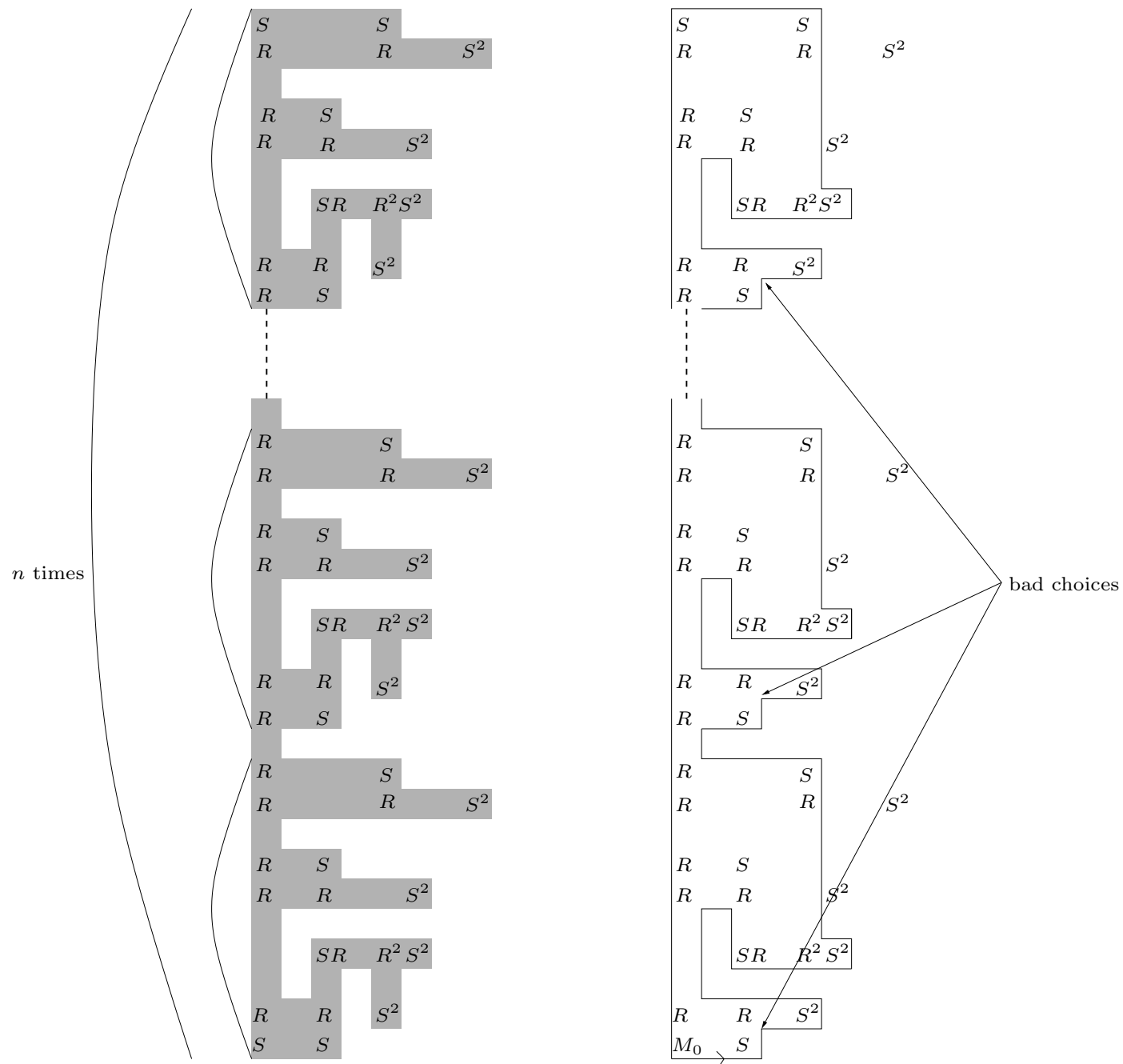

Fig. 9. The set (on the left) takes $\Theta\left(2^{n}\right)$ time to be reconstructed with Algorithm 1. The closed-line (on the right) is the first closed line given by the algorithm, but not all the points have been used, so the the previous choice must be changed.

Intuitively $S U(y)$ and $R U(y)$ are the set of abscissas of the salient and reentrant points whose corresponding corners are directed up.

We will prove that the functions $x_{\min }$ and $x_{\max }$ only depend on $\mathcal{S}_{I}(E)$ and $\mathcal{R}_{I}(E)$. For this we will give a definition of $x_{\min }(y), x_{\max }(y)$ by recurrence on $y$. For $y<\min _{\left(x, y^{\prime}\right) \in E}\left(y^{\prime}\right)$ we have $x_{\min }(y)=+\infty$ and $x_{\max }(y)=-\infty$. Suppose we know $x_{\min }\left(y^{\prime}\right), x_{\max }\left(y^{\prime}\right)$ until $y^{\prime} \leq y-1$. Then we can compute $S U(y-1)$, $R U(y-1)$ from these values. To compute $x_{\min }(y)$, we consider four cases (see Figure 10):

- If $\left(S U(y-1)=\varnothing\right.$ or $\left.x_{\min }(y) \neq \min (S U(y-1))\right)$ and $(S(y)=\varnothing$ or $\left.\min S(y) \geq x_{\min }(y)\right)$ then $x_{\min }(y)=x_{\min }(y-1)$.

- If $S U(y-1) \neq \varnothing, R U(y-1) \neq \varnothing$ and $x_{\min }(y-1)=\min (S U(y-1))$ then $x_{\min }(y)=\min R U(y-1)$. 
- If $S(y) \neq \varnothing$ and $\min S(y)<x_{\min }(y)$ then $x_{\min }(y)=\min S(y)$.

- If $S U(y-1) \neq \varnothing$ and $R U(y-1)=\varnothing$ then $x_{\min }(y)=\min S(y)$.

Similar considerations permit to compute $x_{\max }(y)$. So the functions $x_{\min }$ and $x_{\max }$ only depend on $\mathcal{S}_{I}(E)$ and $\mathcal{R}_{I}(E)$ which proves the proposition.

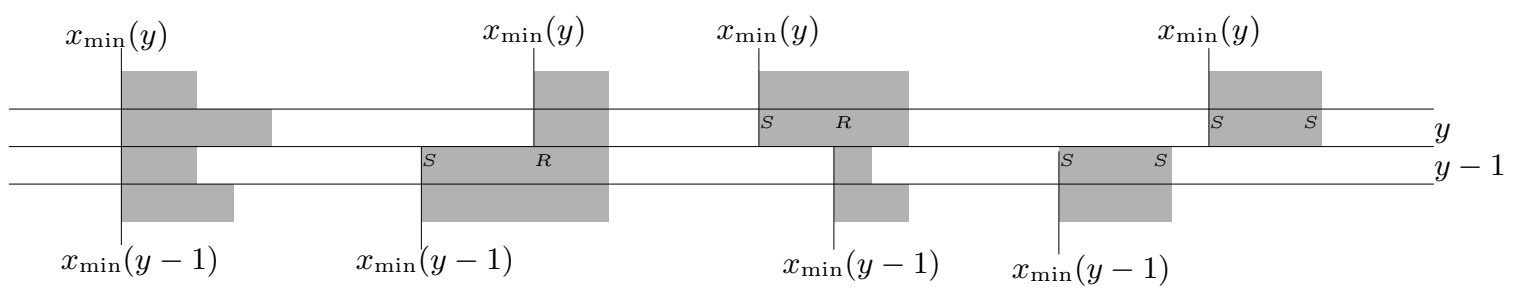

Fig. 10. The four cases in the proof of Proposition 16

Remark 17 This proposition becomes false, if the sets of salient and reentrant points are considered instead of the multisets. (see last example of Figure 7)

\subsection{Characterization of $H V$-convex 8-connected sets by Inner Salient Points}

We will see in this paragraph that the 8-connected HV-convex sets are completely characterized by only their salient inner points without multiplicity.

Proposition 18 Let $E$ and $F$ be two 8-connected $H V$-convex sets then $\operatorname{supp}\left(\mathcal{S}_{I}(E)\right)=$ $\operatorname{supp}\left(\mathcal{S}_{I}(F)\right)$ implies $E=F$.

PROOF. Let $S=\operatorname{supp}\left(\mathcal{S}_{I}(E)\right)=\operatorname{supp}\left(\mathcal{S}_{I}(F)\right)$. For any $M \in \mathbb{Z}^{2}$ we define:

$$
\begin{aligned}
& R_{0}(M)=\left\{(x, y) \in \mathbb{Z}^{2} / x \leq x_{M} \text { and } y \leq y_{M}\right\}, \\
& R_{1}(M)=\left\{(x, y) \in \mathbb{Z}^{2} / x \geq x_{M} \text { and } y \leq y_{M}\right\}, \\
& R_{2}(M)=\left\{(x, y) \in \mathbb{Z}^{2} / x \geq x_{M} \text { and } y \geq y_{M}\right\}, \\
& R_{3}(M)=\left\{(x, y) \in \mathbb{Z}^{2} / x \leq x_{M} \text { and } y \geq y_{M}\right\} .
\end{aligned}
$$

If $R_{0}(M) \cap E \neq \emptyset$ then we see that the lexicographical minimum of $R_{0}(M) \cap E$ is in $S$. As $S \subset E$ we have $R_{0}(M) \cap S \neq \emptyset \Longrightarrow R_{0}(M) \cap E \neq \emptyset$. In the same way we can prove that for any $i \in\{0, \ldots, 3\}$

$$
R_{i}(M) \cap S \neq \emptyset \Longrightarrow R_{i}(M) \cap E \neq \emptyset .
$$

In [2] it is proved that any $\mathrm{HV}$-convex and 8-connected set $E$ is Q-convex along the horizontal and vertical directions which means that

$$
E=\left\{M \in \mathbb{Z}^{2}: \forall i \in\{0, \ldots, 3\} R_{i}(M) \cap E \neq \emptyset\right\}
$$


and so by (8)

$$
E=\left\{M \in \mathbb{Z}^{2}: \forall i \in\{0, \ldots, 3\} R_{i}(M) \cap S \neq \emptyset\right\}
$$

which only depends on $S$.

Remark 19 The definition of the salient points in [3] is equivalent with the inner salient/reentrant points for HV-convex 8-connected sets, but they do not correspond for general sets.

\section{Acknowledgements}

Acknowledgments are due to so regretted Alberto Del Lungo for pointing Proposition 16 to us.

\section{References}

[1] J.-P. Braquelaire, L. Brun, and A. Vialard. Inter-pixel euclidean paths for image analysis. In S. Miguet, A. Montanvert, and S. Ubedan, editors, Disc. Geom. for Comp. Imag. (DGCI'96), volume 1176 of Lecture Notes in Computer Science, pages 193-204. Springer, 1996.

[2] S. Brunetti and A. Daurat. An algorithm reconstructing convex lattice sets. Theoret. Comput. Sci., 304(1-3):35-57, 2003.

[3] A. Daurat. Salient points of Q-convex sets. Int. J. Pattern Recognition \&6 Artificial Intelligence, 15(7):1023-1030, 2001.

[4] A. Rosenfeld. Digital topology. Amer. Math. Monthly, 86(8):621-630, 1979.

[5] A. Rosenfeld. Picture Languages. Academic Press, 1979.

[6] J. Serra. Image analysis and mathematical morphology. Academic Press, 1982. 